\title{
FIXED POINT THEOREMS FOR NONLINEAR NONEXPANSIVE AND GENERALIZED CONTRACTION MAPPINGS
}

\author{
W. A. KIRK
}

\begin{abstract}
Let $X$ be a reflexive Banach space, $H$ a closed convex subset of $X$, and let $K$ be a nonempty, bounded, closed and convex subset of $H$ which possesses normal structure. If $T: K \rightarrow H$ is nonexpansive and if $T: \partial_{H} K \rightarrow K$ where $\partial_{H} K$ denotes the boundary of $K$ relative to $H$, then $T$ has a fixed point in $K$. This result generalizes an earlier theorem of the author, and a more recent theorem of F. E. Browder. An analogue is given for generalized contraction mappings in conjugate spaces.
\end{abstract}

1. Introduction. In [13] we proved that if $K$ is a nonempty, bounded, closed and convex subset of a reflexive Banach space, and if $K$ possesses "normal structure" (defined below), then every nonexpansive mapping $T$ of $K$ into $K$ has a fixed point. This result, also proved independently by F. E. Browder [4] and D. Göhde [11] (in uniformly convex spaces), initiated rather extensive study of fixed point theory for nonlinear nonexpansive operators in Banach spaces, including applications to the study of nonlinear equations of evolution by Browder [5] and to certain nonlinear functional equations (see Browder and Petryshyn [8], Kolomý [16], Srinivasacharyulu [21]).

In this paper we modify the approach of [13] to treat the following problem: Given closed and convex subsets $K$ and $H$ of a Banach space $X$ such that $K \cap H \neq \varnothing$ and an operator $T: K \rightarrow X$ such that (i) $T: K \cap H \rightarrow H$ and (ii) $T: \partial_{I I} K \rightarrow K$ (where $\partial_{H} K$ denotes the relative boundary of $K \cap H$ in $H$ ), when does $T$ have a fixed point? This kind of problem has been of particular interest in the case where the operator $T$ is completely continuous, $H$ is the positive cone of $X$, and the fixed points of $T$ correspond to positive solutions of a differential equation (for example, see [17]). A standard approach is to use the technique of "radial projection" to associate with $T$ an operator $B$ which is also completely continuous, has the same fixed points as $T$, and maps the intersection of $H$ with the ball $K:\|x\| \leqq R$ into itself, thus permitting application of the classical Schauder Theorem [19]. Such an approach, however, is not suitable for our purposes because we consider mappings of nonexpansive type. Since radial projection is in general not nonexpansive (see [9]), the associated operator $B$ need not be nonexpansive and one cannot obtain a fixed point by direct application of the theorem of [13]. 
Before stating our results we establish relevant notation and definitions.

A mapping $T$ of a subset $K$ of a Banach space $X$ into $X$ is called nonexpansive if $\|T x-T y\| \leqq\|x-y\|$ for all $x, y \in K$.

For a subset $S$ of a Banach space $X$, the symbol $\delta(S)$ denotes the diameter of $S$-i.e.,

$$
\delta(S)=\sup \{\|x-y\| ; x, y \in S\} .
$$

The notation $U(z ; r)$ is used to denote the spherical neighborhood of $z$ of radius $r>0$ :

$$
U(z ; r)=\{x \in X:\|z-x\|<r\} .
$$

Similarly,

$$
\bar{U}(z ; r)=\{x \in X:\|z-x\| \leqq r\} .
$$

The concept of normal structure, due to Brodskii and Milman [3], plays a key role in our approach. A bounded convex set $K$ in a Banach space $X$ is said to have normal structure if for each convex subset $S$ of $K$ which contains more than one point, there is a point $x \in S$ which is a nondiametral point of $S$ (i.e., $\sup \{\|x-y\|: y \in S\}<$ $\delta(S))$. Compact convex sets possess normal structure ([3], [10, Lemma 1]) as do all bounded convex subsets of uniformly convex spaces. (For a comparison of normal structure and uniform convexity, see BelluceKirk-Steiner [2]. The concept has also been studied by Gossez and Lami Dozo [12].)

We wish to thank the referee for his suggestions, particularly for pointing out the corollary to Theorem 3.1.

2. A fixed point theorem for nonexpansive mappings. For $H$ and $K$ subsets of $X$, we use the symbol $\partial_{H H} K$ to denote the boundary of $K$ relative to $H$ : Thus, letting $H-K$ denote the points of $H$ which are not in $K$, if $K$ is closed,

$$
\partial_{H} K=\{z \in K: U(z ; r) \cap(H-K) \neq \varnothing \text { for each } r>0\} .
$$

Theorem 2.1. Let $X$ be a reflexive Banach space, $H$ a closed convex set in $X$, and $K$ a nonempty, bounded, closed, convex subset of $H$ which possess normal structure. If $T: K \rightarrow H$ is nonexpansive, and if $T: \partial_{H} K \rightarrow K$, then $T$ has a fixed point in $K$.

The above theorem immediately reduces to our theorem of [13] upon taking $H=K$. A more interesting consequence of this theorem arises from taking $H=X$ : 
Corollary. Let $K$ be a bounded closed convex subset of a reflexive Banach space $X$ and suppose $K$ possesses normal structure. If $T: K \rightarrow X$ is nonexpansive, and if $T$ maps the boundary of $K$ into $K$, then $T$ has a fixed point in $K$.

Browder first obtained the above result [6, Theorem 3] for $K$ a bounded closed convex set in a uniformly convex space with the additional assumption that $T$ is defined on an open convex set $G \supset K$ with $\operatorname{dist}(K, X-G)>0$. Subsequently Browder [7] and Nussbaum [18] have removed this assumption (in a uniformly convex setting) while proving more general results, a fact which is significant because in general one may not enlarge the domain of nonexpansive mapping [20].

Proof of Theorem 2.1. Let $\mathscr{T}$ be the family of all closed convex subsets of $H$ such that for $F \in \mathscr{T}, F \cap K \neq \varnothing$ and $T: F \cap K \rightarrow F$. Since $H \in \mathscr{T}, \mathscr{T} \neq \varnothing$. Let $\left\{F_{\alpha}\right\}$ be a descending chain of sets of $\mathscr{T}$, and let $F=\bigcap_{\alpha} F_{\alpha}$. Note that $F \cap K$ is nonempty, since each of the sets $F_{\alpha} \cap K$ is a nonempty weakly compact subset of $X$. Also, since $T: F_{\alpha} \cap K \rightarrow F_{\alpha}$ for each $\alpha$, clearly $T: F \cap K \rightarrow F$. Since $F$ is closed and convex, $F \in \mathscr{G}$, and therefore by Zorn's Lemma, $\mathscr{T}$ has a minimal element.

Letting $F$ be such a minimal element of $\mathscr{T}$, first note that we may sssume $\partial_{F} K \neq \varnothing$, for otherwise $F \subset K$ and $T: F \cap K \rightarrow F$ would imply $T: F \rightarrow F$. The existence of a fixed point would then follow from the theorem of [13].

Now we assume $\hat{o}(F \cap K)>0$ and obtain a contradiction. Let $\hat{o}=\delta(F \cap K)$. Since $K$ possesses normal structure, there exists a point $c \in F \cap K$ such that

$$
\sup \{\|c-z\|: z \in F \cap K\}=r<\delta \text {. }
$$

Let

$$
C=\{x \in X: F \cap K \subset \bar{U}(x ; r)\} .
$$

It is easily seen that $C$ is closed and convex and, since $c \in F \cap C$, $(F \cap C) \cap K \neq \varnothing$. Also there exist points $x, y \in F \cap K$ such that $\|x-y\|>r$. Such points cannot be elements of $C$ and therefore $F \cap C$ is a proper subset of $F$. We complete the proof by showing $F \cap C \in \mathscr{T}$. Since we have already seen that $(F \cap C) \cap K \neq \varnothing$, we need only show that $T:(F \cap C) \cap K \rightarrow F \cap C$.

Suppose $z \in(F \cap C) \cap K$. Let

$$
W=\bar{U}(T z ; r) \cap F .
$$

If $W \in \mathscr{I}$, then since $W \subset F$ and $F$ is minimal, $W=F$. This implies 


$$
F \cap K \subset F \subset \bar{U}(T z ; r)
$$

and hence $T z \in C$. Since $T: F \cap K \rightarrow F$, this in turn yields $T z \in F \cap C$. Therefore $T:(F \cap C) \cap K \rightarrow F \cap C$ if $W \in \mathscr{J}$ for every $z \in(F \cap C) \cap K$. We complete the proof by showing this to be the case.

First suppose $x \in W \cap K$. Then $x \in F \cap K$ so $\|x-z\| \leqq r$ (because $z \in C)$. Hence $\|T x-T z\| \leqq r$ and $T x \in \bar{U}(T z ; r)$. But $z \in W \cap K$ also implies $x \in F \cap K$ and hence $T x \in F$. Therefore $T x \in \bar{U}\left(T z ; \gamma^{2}\right) \cap F=$ $W$, i.e., $T: W \cap K \rightarrow W$.

Finally, since $\partial_{F} K \neq \varnothing$, it follows that $W \cap K \neq \varnothing$. To see this, note that if $y \in \partial_{F} K$ then $y \in F \cap K$ and $\|y-z\| \leqq r$, which implies $\|T y-T z\| \leqq r$ and therefore $T y \in W$. But also $\partial_{F} K \subset \partial_{I I} K$ implies $T y \in K$; hence $T y \in W \cap K$ and $W \cap K \neq \varnothing$.

This completes the proof that $F \cap C \in \mathscr{T}$, contradicting the assumption $\delta(F \cap C)>0$. Thus $\delta(F \cap C)=0$ and $F \cap C$ consists of a single point which, because $T: \partial_{F} K \rightarrow K$, is fixed under $T$.

3. Generalized contraction mappings. In this section we give an analogue of Theorem 2.1 for the class of generalized contraction mappings studied in $[14,15]$. With $X$ a Banach space, and $K \subset X$, a mapping $T: K \rightarrow X$ is called a generalized contraction mapping if for each $x \in K$ there is a number $\alpha(x)<1$ such that

$$
\|T x-T y\| \leqq \alpha(x)\|x-y\| \quad \text { for each } y \in K \text {. }
$$

It was noted in Belluce-Kirk [1] that mappings of this type provide an example of a class of mappings with "diminishing orbital diameters"; thus fixed point theorems proved in [1] apply to this class of mappings. In [15] it is shown that if $A$ is a bounded open convex subset of $X$ and if $F: A \rightarrow X$ is continuously Fréchet differentiable on $A$, then $F$ is a generalized contraction mapping on $A$ if and only if for each $x_{0} \in A$ the norm of the Fréchet derivative $F_{x_{0}}^{\prime}$ of $F$ at $x_{0}$ is less than one. It is also shown that if $K$ is a $w^{*}$ compact convex subset of a conjugate Banach space $X$ and if $T: K \rightarrow K$ is a generalized contraction mapping, then $T$ has a fixed point in $K$. This result may be generalized as follows:

THEOREM 3.1. Let $X$ be a conjugate Banach space, $H$ a convex $w^{*}$-closed subset of $X$, and $K$ a nonempty convex $w^{*}$-compact subset of $H$. If $T: K \rightarrow H$ is a generalized contraction mapping on $K$, and if $T: \partial_{H} K \rightarrow K$, then $T$ has a fixed point in $K$.

Proof. As in the proof of Theorem 2.1, obtain a $w^{*}$-compact convex set $F$ minimal with respect to the properties $F \cap K \neq \varnothing$ and 
$T: F \cap K \rightarrow F$. As before, it may be assumed that $\partial_{F}(K) \neq \varnothing$ (otherwise $F \subset K$ and existence of a fixed point follows from Theorem 1.1 of [15]).

The argument parallels that of Theorem 2.1 upon obtaining a point $c \in F \cap K$ such that

$$
\sup \{\|c-z\|: z \in F \cap K\}<\delta \text {. }
$$

Such a point can be obtained by letting $x \in \partial_{F} K$, noting that $T x \in F \cap K$, and using the procedure of the proof of Theorem 1.1 of [15] to show that $T x$ has the property specified for $c$ in (1). Specifically, one can show that if $\delta=\delta(F \cap K)>0$ then for the number $\alpha(x)<1$ associated with $T$ by definition, one has

$$
\bar{U}(T x ; \alpha(x) \delta) \cap F \in \mathscr{G}
$$

which implies

$$
\sup \{\|T x-z\|: z \in F \cap K\} \leqq \alpha(x) \delta=r<\delta .
$$

Then letting $T x=c$, define the set $C$ as in Theorem 2.1 and observe that

$$
C=\bigcap_{x \in F \cap K} \bar{U}(x ; r)
$$

Thus $C$ is $w^{*}$-compact and convex. This and the fact that the set $W$ defined later in the argument is also $w^{*}$-compact and convex, enables one to complete the proof precisely as in Theorem 2.1. We omit the details.

Cozollary. If $X$ is a conjugate Banach space and $H$ is a closed convex subset of $X$ of which every intersection with a $w^{*}$-compact set is $w^{*}$-compact (e.g. $H=X$ ), and if $T: H \rightarrow H$ is a generalized contraction mapping on $H$, then $T$ has a fixed point in $H$.

Proof. Let $x \in H$ and let

$$
K=H \cap \bar{U}\left(x ; \frac{\|x-T x\|}{1-\alpha(x)}\right) .
$$

Then $T: \partial_{H} K \rightarrow K$ and by Theorem 3.1, $T$ has a fixed point in $H$.

\section{REFERENCES}

1. L. P. Belluce and W. A. Kirk, Fixed point theorems for certain classes of nonexpansive mappings, Proc. Amer. Math. Soc., 20 (1969), 141-146.

2. L. P. Belluce, W. A. Kirk, and E. F. Steiner, Normal structure in Banach spaces, Pacific J. Math., 26 (1968), 433-440. 
3. M. S. Brodskii and D. P. Milman, On the center of a convex set, Dokl. Acad. Nauk SSSR 59 (1948), 837-840.

4. F. E. Browder, Nonexpansive nonlinear operators in a Banach space, Proc. Nat. Acad. Sci., 54 (1965), 1041-1044.

5. - Periodic solutions of nonlinear equations of evolution in infinite dimensional spaces, Lecture Series in Differential Equations, University of Maryland, 1966 (AFOSR 65-1886).

6. Nonlinear mappings of nonexpansive and accretive type in Banach spaces, Bull. Amer. Math. Soc., 73 (1967), 875-881.

7. - Semicontractive and semiaccretive nonlinear mappings in Banach spaces, Bull. Amer. Math. Soc., 74 (1968), 660-665.

8. F. E. Browder and W. V. Petryshyn, The solution by iteration of nonlinear functional equations in Banach spaces, Bull. Amer. Math. Soc., 72 (1966), 571-575.

9. D. G. De Figueiredo and L. A. Karlovitz, On the radial projection in normed spaces, Bull. Amer. Math. Soc., 73 (1967), 364-368.

10. R. De Marr, Common fixed points for commuting contraction mappings. Pacific J. Math., 13 (1963), 1139-1141.

11. D. Göhde, Zum Prinzip der kontraktiven Abbildung, Math. Nachr., 30 (1965), $251-258$

12. J. P. Gossez and E. Lami Dozo, Structure normale et base de Schauder. Bull. de l'Acad. royale de Belgique (5e Serie) $\mathbf{5 5}$ (1969), 673-681.

13. W. A. Kirk, A fixed point theorem for mappings which do not increase distances, Amer. Math. Monthly, 72 (1965), 1004-1006.

14. - On nonlinear mappings of strongly semicontractive type, J. Math Anal. Appl., 27 (1969), 409-412.

15. - Mappings of generalized contractive type, J. Math. Anal. Appl., 32 (1970), $567-572$.

16. J. Kolomý, Some existence theorems for nonlinear problems, Comment. Math. Univ. Carolinae 7 (1966), 207-212.

17. M. A. Krasnosel'skii, Positive solutions of operator equations, Groningen, P. Nordhoff Ltd., 1964.

18. R. D. Nussbaum, The fixed point index and asymptotic fixed point theorems for k-set-contractions, Bull. Amer. Math. Soc., 75 (1969), 490-495.

19. J. Schauder, Der Fixpunktsatz in Funktionalraïmen, Studia Mathematika, 2 (1930), 171-180.

20. O. Schonbeck, On the extension of Lipshitz maps, Ark. Mat., 7 (1967), 201-209.

21. K. Srinivasacharyulu, On some non-linear problems, Canad. J. Math. 20 (1968), $394-397$.

Received March 20, 1970 and in revised form September 2, 1970. Research supported by National Science Foundation grants GP-8367 and GP-18045.

The University of Iowa. 